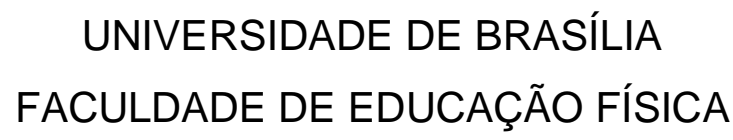

COMPARAÇÃO DE VARIÁVEIS FÍSICAS E DOMÍNIOS DE QUALIDADE DE VIDA ENTRE INDIVÍDUOS AVALIADOS PELO MÉTODO FUNCTIONAL MOVEMENT SCREEN

(FMS)

Matheus Almeida Trindade

BRASÍLIA 


\title{
COMPARAÇÃO DE VARIÁVEIS FÍSICAS E DOMÍNIOS DE QUALIDADE DE VIDA ENTRE INDIVÍDUOS AVALIADOS PELO MÉTODO FUNCTIONAL MOVEMENT SCREEN
} (FMS)

\author{
Matheus Almeida Trindade
}

\begin{abstract}
Dissertação apresentada à Faculdade de Educação Física da Universidade de Brasília, como requisito parcial para obtenção do grau de Mestre em Educação Física.
\end{abstract}

ORIENTADOR: PROF. DR. RODRIGO LUIZ CARREGARO 
Ficha catalográfica elaborada automaticamente, com os dados fornecidos pelo(a) autor(a)

Almeida Trindade, Matheus COMPARAÇÃO DE VARIÁVEIS FÍSICAS E DOMÍNIOS DE QUALIDADE DE VIDA ENTRE INDIVÍDUOS AVALIADOS PELO MÉTODO FUNCTIONAL MOVEMENT SCREEN (FMS) / Matheus Almeida Trindade; orientador Rodrigo Luiz Carregaro. -- Brasilia, 2015. $50 \mathrm{p}$.

Dissertação (Mestrado - Mestrado em Educação Física) -- Universidade de Brasilia, 2015.

1. Educação Física. 2. Funcionalidade. 3. Avaliação Funcional. I. Luiz Carregaro, Rodrigo, orient. II. Título. 


\section{Sumário}

1 INTRODUÇÃO

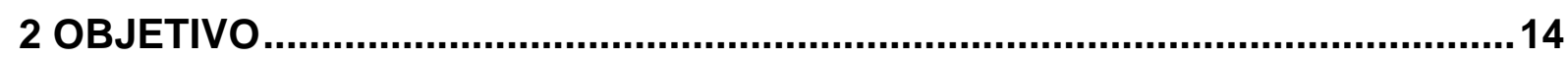

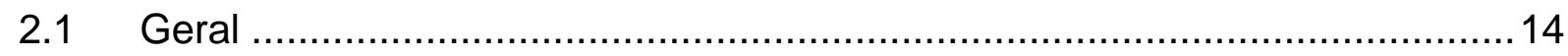

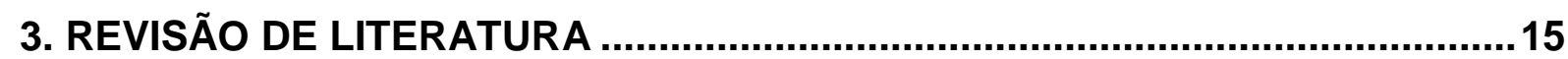

3.1 FMS no contexto da previsão de lesões musculoesqueléticas .....................15

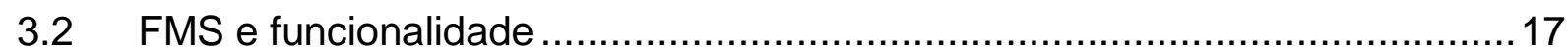

3.3 FMS no contexto do equilíbrio e propriocepção ....................................... 19

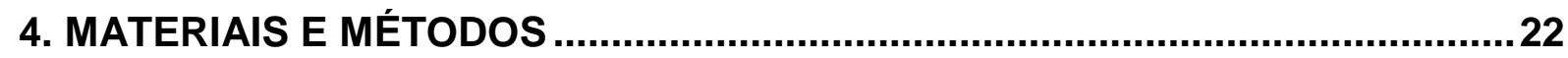

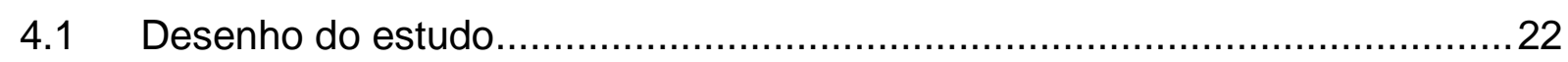

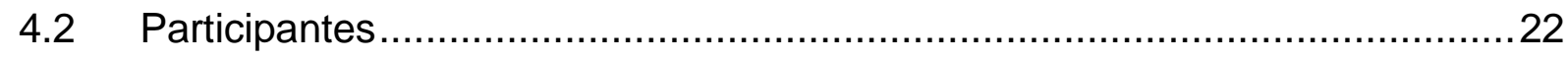

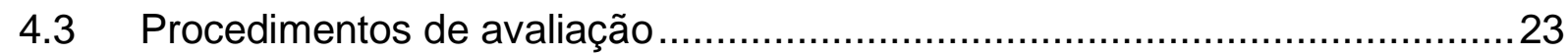

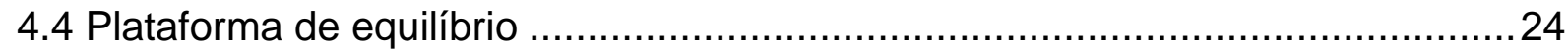

4.5 Questionário internacional de atividade física (IPAQ) ......................................25

4.6 Questionário de qualidade de vida (SF-36) ................................................26

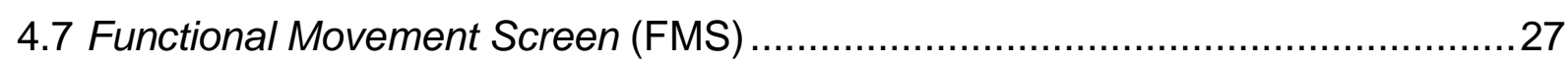

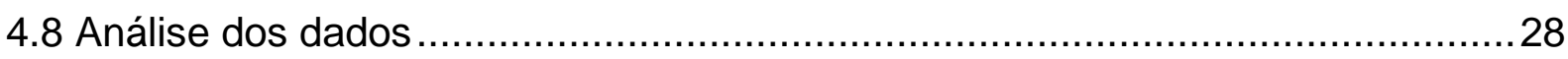

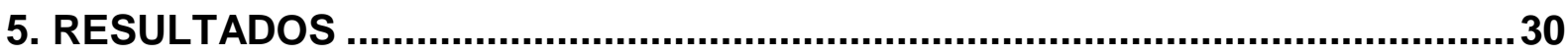

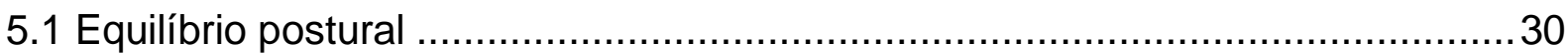

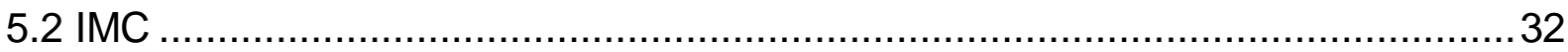

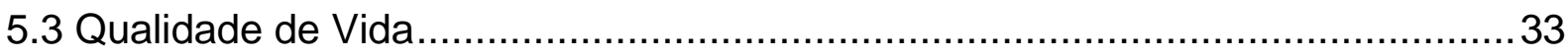

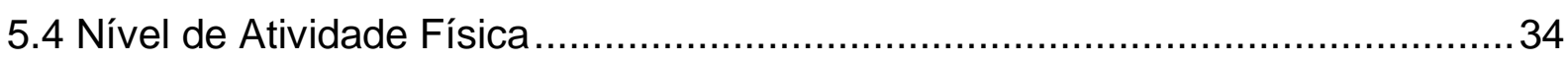

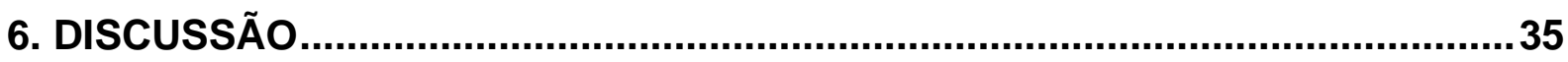

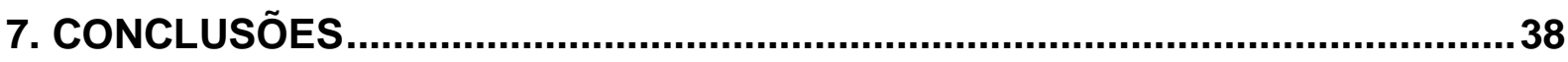

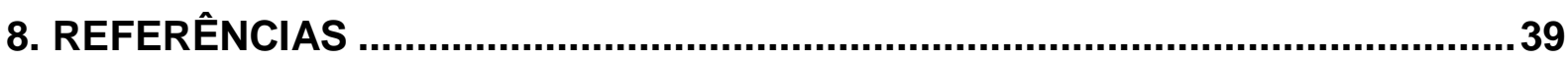

ANEXO I

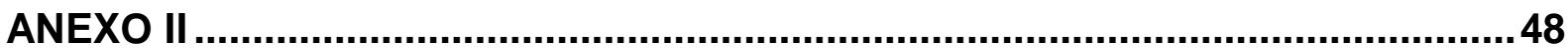

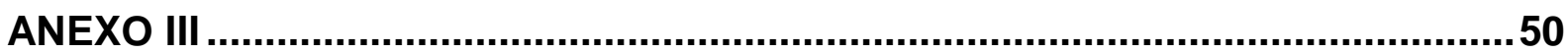




\section{LISTA DE TABELAS}

Tabela 1 Caracterização da amostra .23

Tabela 2. Domínios de qualidade de vida dos participantes do estudo, com base no questionário SF-36. 33

Tabela 3. Nível de atividade física e classificação do FMS. .34 


\section{LISTA DE FIGURAS}

Figura 1 llustração das sete posturas do FMS (Adaptado de (Butler, Contreras et al.

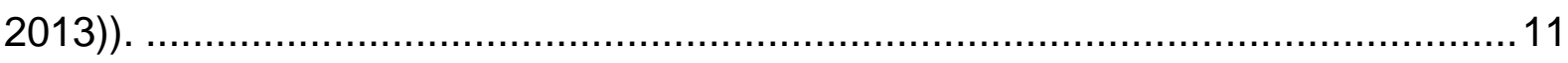

Figura 2. Ilustração que representa o desenho do estudo.....................................24 


\section{LISTA DE SIGLAS}

ASL - Índice de Oscilação Corporal em Apoio Unipodal

ASLR - Active Straight-Leg Raise

BBS - Biodex Balance System

CEP - Comitê de Ética em Pesquisa

DS - Deep Squat

FMS - Functional Movement Screen

HS - Hurdle Step

ILL - Inline Lunge

IPAQ - International Physical Activity Questionnaires

LCA - Ligamento Cruzado Anterior

RS - Rotary Stability

SM - Shoulder Mobility

TCLE - Termo de Consentimento Livre e Esclarecido

TSP - Trunk Stability Pushup

UnB - Universidade de Brasília

FS - Faculdade de Saúde 


\section{RESUMO}

Introdução Entende-se que a avaliação dos movimentos funcionais é uma forma de identificar deficiências de mobilidade e estabilidade, por vezes negligenciados em avaliações clássicas e tradicionais, até mesmo na população assintomática. Nosso estudo é pioneiro ao tentar relacionar a qualidade de vida com os resultados do FMS. Objetivo Avaliar o equilíbrio postural estático e dinâmico, nível de atividade física e a qualidade de vida de universitários jovens e sadios. Métodos Trata-se de um estudo transversal, caracterizado pela aplicação do método FMS em indivíduos jovens e sadios. Os voluntários foram submetidos a testes de equilíbrio e questionários de nível de atividade física e qualidade de vida. Participaram do presente estudo 58 voluntários, de ambos os sexos, universitários, de 18 a 35 anos e sem histórico de trauma ou lesões que pudessem gerar algum impedimento na realização das atividades do trabalho. Resultados $O$ presente estudo demonstrou que o nível de atividade física, domínios de qualidade de vida e índices de equilíbrio unipodal de universitários sadios não discriminaram os grupos $F_{M S}$ e $F M S_{2}$. Considerações finais Tais achados reforçam a importância de se rever o uso do FMS enquanto única estratégia para prevenção de lesões ou análise de desempenho durante atividades físicas e esportivas.

Descritores: Movimento; Funcionalidade; FMS 


\begin{abstract}
Introduction It is understood that the evaluation of functional movements is a way to identify deficiencies of mobility and stability, sometimes neglected in classical and traditional assessments even in the asymptomatic population. Our study is the first to try to relate the quality of life with the results of FMS. Objective To evaluate static and dynamic postural balance, physical activity and quality of life of young students and healthy. Methods This was a cross-sectional study, characterized by the method FMS in young and healthy individuals. The volunteers underwent balance tests and questionnaires level of physical activity and quality of life. The study included 58 volunteers of both sexes, students, 18-35 years and with no history of trauma or injury that could generate some impediment in carrying out work activities. Results The study showed that the level of physical activity, quality of life domains and single leg balance indexes of healthy university did not discriminate the FMS1 and FMS2 groups. Conclusion These findings reinforce the importance of reviewing the use of FMS as the sole strategy for prevention of injury or performance analysis for physical and sporting activities.
\end{abstract}

Key-words: Movement; Functionality; FMS 


\section{INTRODUÇÃO}

O Functional Movement Screen (FMS) foi criado em 1997 e, desde então, tem sido foco de pesquisas científicas (Burton 2011). O FMS é uma ferramenta de avaliação que tem por objetivo avaliar padrões de movimento individual, e propicia um modelo de avaliação em condições dinâmicas e funcionais (Cook, Burton et al. 2006). Três aspectos são inerentes ao FMS: (1) é uma ferramenta de rápida e fácil aplicação em quase qualquer ambiente; (2) pode ser utilizado para identificar indivíduos que estão em risco de lesão, dentro de certos grupos da população; (3) a pontuação da FMS pode melhorar por meio de intervenções (Burton 2011).

Entende-se que a avaliação dos movimentos funcionais é uma forma de identificar deficiências de mobilidade e estabilidade, por vezes negligenciados em avaliações clássicas e tradicionais, até mesmo na população assintomática. O FMS pode ser incluído em uma pré-participação do exame físico, ou pode ser utilizado como uma técnica de avaliação independente para determinar os déficits que podem estar ocultos em avaliações de rotina ou tradicionais (Cook, Burton et al. 2006).

O FMS dispõe de sete (7) posturas (Figura 1): Deep Squat (agachamento profundo que avalia bilateralmente e simetricamente, a funcionalidade dos quadris, joelhos e tornozelos), Hurdle Step (um passo através de um obstáculo, examinando a mecânica da passada), Inline Lunge (mobilidade do quadril e tronco, estabilidade de tornozelo e joelho, e flexibilidade dos isquiotibiais), Shoulder Mobility (mobilidade do ombro bilateralmente), Active Straight-Leg Raise (levantar a perna ativamente em extensão de joelho, determinando a atividade do tendão e flexibilidade gastrocnêmio-sóleo, mantendo ao mesmo tempo uma pélvis estável), Trunk Stability Pushup (estabilidade do tronco, enquanto a simetria de membros superiores é executada), Rotary Stability (teste da estabilidade de rotação, que avalia a estabilidade do tronco enquanto os membros superiores e inferiores estão em movimento combinado). A pontuação de cada teste varia de 0 a 3 de acordo com a qualidade do movimento; as pontuações são combinadas para um escore final que se considera para prever lesões. De acordo com estudos, a classificação igual ou inferior a 14 pontos representa uma maior probabilidade de lesão sem contato em populações fisicamente ativas (Kiesel, Plisky et al. 2007; Chorba, Chorba et al. 2010; 
O'Connor, Deuster et al. 2011; Kazman, Galecki et al. 2013; Garrison, Westrick et al. 2015).

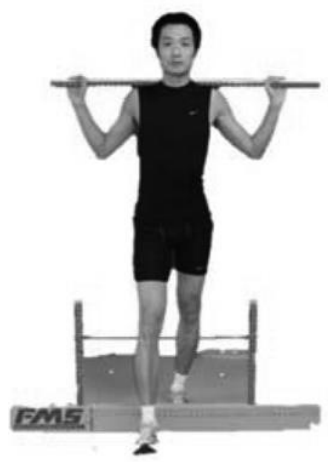

Hurdle Step

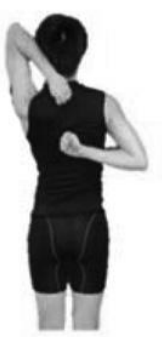

Shoulder Mobility

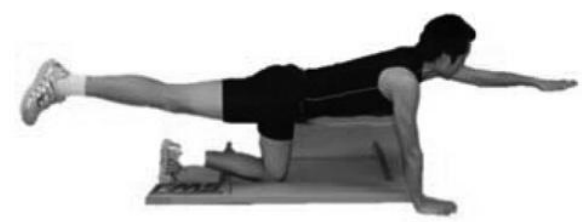

Rotary Stability

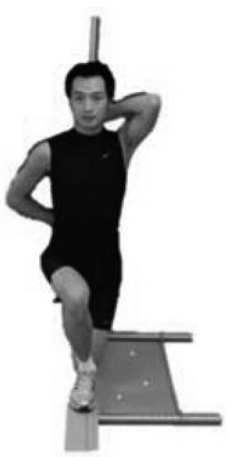

In Line Lunge

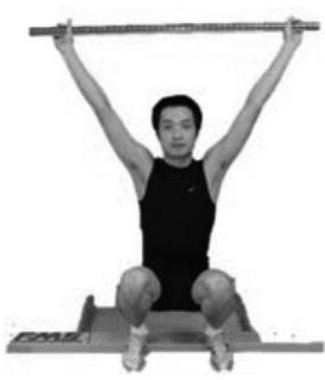

Deep Squat

Figura 1 llustração das sete posturas do FMS (Adaptado de (Butler, Contreras et al. 2013)).

Nosso estudo é pioneiro ao tentar relacionar a qualidade de vida com os resultados do FMS. A expansão do interesse no FMS levou pesquisadores a investigarem com que precisão se pode identificar os indivíduos com alto risco de lesão. A melhor evidência mundial hoje é o escore 2, o que nos levou a pensar, se com os bons resultados dos estudos que mostram a acurácia do FMS em identificar indivíduos com elevado risco de lesão musculoesquelética, se outras variáveis que não apenas as musculares, discriminariam o resultado do FMS (Krumrei, Flanagan et al. 2014).

Em estudo recente, Bodden, Needham e Chockalingam (2013) realizaram um programa de intervenção em atletas de MMA, e obtiveram resultados significantes 
que foram comprovados por meio da pontuação total do FMS no grupo intervenção, comparados ao grupo controle. Tais achados demonstram que o FMS foi sensível aos efeitos de programas de exercício. Vale ressaltar que os atletas mantiveram suas rotinas de treinamento, indicando que o FMS é uma ferramenta de avaliação interessante. No entanto, Kazman et al. (2013) e Li, Wang, Chen e Dai (2015) realizaram estudo no qual concluíram que a consistência interna do FMS ainda é falha, o que justifica novas pesquisas. Ainda, os autores apresentaram indícios de que cada postura seja independente e, portanto, seja melhor trabalhar posturas individualizadas ao invés de uma soma total do método. Tais aspectos demonstram que a aplicabilidade e interpretação dos achados do FMS devem ser estudadas.

Outras pesquisas seguem o caminho da análise da relação do FMS com 0 desempenho esportivo. A exemplo, Okada, Huxel e Nesser (2011) e Parchman e Mcbride (2011) realizaram a comparação do FMS com testes de corrida de agilidade e agachamento, com a justificativa de serem comumente usados para predizer o desempenho esportivo. As conclusões dos autores convergiram no sentido de que o FMS não possuía relação com performance da corrida e agachamento, sendo assim, possivelmente não possuindo relação com performance esportiva.

Em estudos posteriores, houve resultados que corroboram os achados de Okada et al. (2011) e Parchman e McBride (2011). Entretanto, Bodden, Needham e Chockalingam (2013) e Chapman, Laymon e Arnold (2014) chegaram a conclusões diferenciadas, com base na avaliação de atletas profissionais. Os autores realizaram testes específicos de cada esporte, e notaram a possibilidade do FMS servir como um auxiliar da preparação física desses atletas. Dentre os trabalhos citados, Chapman realizou estudo com maior amostra, sendo um total de 121 atletas da elite do atletismo norte americano.

O FMS tem por característica a praticidade de aplicação, o baixo custo e pouco tempo necessário para sua realização, que é em média de 25 minutos por sujeito, de acordo com estudo prévio realizado. Único material utilizado para a aplicação do método, o kit do programa custa menos de mil reais, sendo utilizado para todos os voluntários.

Por fim, vale destacar que estudos mais recentes buscam a compreensão da relação entre o FMS e outros domínios, sejam eles físicos ou biopsicossociais. A exemplo, Bradley e Esformes (2014) demonstraram que a qualidade da respiração 
diafragmática possui uma relação com o movimento funcional , indicando que o FMS pode ser uma ferramenta útil no contexto da prevenção e reabilitação. 


\section{OBJETIVOS}

\section{$2.1 \quad$ Geral}

Comparar o equilíbrio postural estático e dinâmico, nível de atividade física e a qualidade de vida de universitários jovens e sadios avaliados pelo método FMS e classificados com base em escore total do método ( $\leq 14$ e $>14)$. 


\section{REVISÃO DE LITERATURA}

\subsection{FMS no contexto da previsão de lesões musculoesqueléticas}

Usualmente, programas que são prescritos para ganhos de força e condicionamento físico tem a capacidade de oferecer ganhos na mobilidade, velocidade e força, sem considerar a perfeição ou eficiência do movimento/técnica do exercício. Um exemplo pode ser uma pessoa que tem uma média superior no número de abdominais durante um teste, mas é executado de modo ineficientemente com compensações e movimento da parte superior do tronco e coluna cervical. Por outro lado, outros indivíduos também podem apresentar uma média superior no número de abdominais, mas executam perfeitamente a técnica, sem a utilização de compensações corporais. Esses dois indivíduos podem ser considerados "acima da média" quando não consideradas as ineficiências corporais. No entanto, uma importante questão se refere às presenças de deficiências no movimento e se as mesmas são notadas em seus movimentos funcionais. Além disso, questiona-se se o desempenho desses indivíduos devem ser considerados como iguais.

Nos últimos anos, profissionais que atuam com reabilitação têm buscado alternativas à avaliação tradicional, para uma abordagem de avaliação funcional, que integra princípios da facilitação neuromuscular proprioceptiva (FNP), sinergia muscular e aprendizado motor (Cook, Burton et al. 2006).

Criado em 1997 por Cook, Burton e Hoogenboom, o FMS surgiu como opção de análise da "qualidade" do movimento. Desse modo, o FMS pode ser caracterizado como uma análise do movimento sob o ponto de vista funcional, tendo como base os desequilíbrios musculares e compensações que podem ocasionar lesões musculoesqueléticas. Como diferencial das intervenções que tem por objetivo diminuir as lesões, aumentar o desempenho e melhorar a qualidade de vida, o FMS fornece uma análise diferenciada dos movimentos. Tal aspecto é reforçado pela característica do processo de avaliação do FMS, que ocorre de forma dinâmica e contempla uma avaliação global do corpo humano. Esse fator propicia aos 
profissionais que atuam na área da cinesiologia uma nova maneira de abordar o paciente, deixando de se avaliar e desenvolver prioritariamente o movimento específico de certa atividade, para se dar ênfase primeiramente ao movimento tido como funcional e mais global (Cook, Burton et al. 2006).

Devido à possibilidade aventada pelos criadores do FMS em prever lesões, esportes e atividades nos quais há grandes investimentos financeiros, principalmente nos Estados Unidos, começaram a utilizar o método com o intuito de reduzir os gastos e impactos das lesões no desempenho dos atletas. Entre os exemplos estão o hockey, futebol americano, basquete, baseball e militares (Sorenson 2009; Lisman, O'Connor et al. 2013; Parenteau-G, Gaudreault et al. 2014).

Kiesel, Plisky e Voight (2007), em estudo com jogadores profissionais de futebol americano, tiveram por objetivo determinar a relação entre a pontuação do FMS e a probabilidade de lesões graves, caracterizadas por afastamento mínimo de três semanas. Os autores verificaram que houve uma relação entre o FMS e as presença das lesões, indicando que indivíduos com escores do FMS iguais ou menores a 14 apresentavam maior índice de lesões ao longo da temporada. No mesmo ano, Peate et al. (2007) aplicaram em uma população de bombeiros, um treinamento com intenção de diminuir as lesões comumente encontradas nessa população. Por meio dos resultados do FMS, realizou-se treinamento de estabilidade muscular e obteve-se diminuição de $62 \%$ da perda de tempo devido às lesões e $42 \%$ no número de lesões quando comparado com um grupo controle.

Assim como usado em adultos, o FMS também começou a ser usado em diferentes populações. Chorba et al. (2010) avaliou atletas colegiais e teve por objetivo determinar se movimentos compensatórios predispunham lesões e se o FMS poderia predizer tais lesões. Os resultados mostraram que ambas as hipóteses tiveram resposta afirmativa, o que já era esperado tendo em vista que o FMS é um método de avaliação da qualidade do movimento.

No ano de 2011, em um estudo com homens candidatos a oficial da marinha americana, realizou-se análise para verificar a incidência de lesões ao longo do período de treinamentos obrigatórios dos candidatos. Juntamente com este objetivo, os autores procuraram determinar um escore preditivo de lesão. Em ambos os 
estudos, de 2010 e 2011, o FMS foi sensível e teve capacidade preditora das lesões, corroborando estudos prévios (O'Connor, Deuster et al. 2011).

Butler et al. (2013) desenvolveram estudo semelhante, no qual analisaram a previsão de lesões por meio do FMS. Os autores realizaram estudo com grupo de bombeiros e verificaram a mesma faixa de valores do escore total (menor ou igual a 14, para maior risco de lesão). Fato interessante foi que também foi verificado que o deep squat e o push-up possuíam, estatisticamente, maior poder de predição em relação aos outros movimentos quando analisados individualmente.

Garrison et al. (2015) ratificaram o valor preditivo do FMS encontrado nos outros artigos, e concluíram que a combinação do resultado do FMS com o histórico de lesão aumentou o poder de previsibilidade de lesões. Alguns estudos demonstraram valores diferentes, como Letafatkar et al. (2014) que realizaram estudo com estudantes fisicamente ativos (50\% homens e 50\% mulheres), e chegaram a mesma conclusão dos estudos prévios quanto a relação existente entre o resultado do FMS e lesão, porém, seus achados demonstraram um escore $\leq 16$ com maior valor preditivo para as lesões.

Vale salientar que o FMS não é aplicável a todo tipo de atleta ou esporte. Em estudo de 2014, durante a pré-temporada de jogadores de hockey da categoria júnior, foi realizada uma triagem dos atletas na tentativa de prever lesões para o decorrer da temporada. Concluiu-se que o FMS não obteve resultados significantes como método preditor de lesão para esta população. Porém, segundo relato dos próprios autores, todas as lesões foram por contato natural do esporte, o que não é o objetivo do FMS, por se tratar de fato inesperado e, portanto, imprevisível. Nesse caso, o objetivo do FMS é analisar déficits de movimento para evitar problemas crônicos e prever lesões sem contato (Dossa, Cashman et al. 2014).

\section{2 $\quad$ FMS e funcionalidade}

No contexto da função, uma importante aplicação do FMS está relacionada ao acompanhamento de treinamentos físico-funcionais. Cowe (2010) avaliou os benefícios da yoga em bombeiros fisicamente ativos e não praticantes da mesma. Para isso, realizou dez sessões tradicionais de yoga que incluíam respiração, 
postura e relaxamento, obtendo resultados significantes na melhora do estresse, flexibilidade de tronco e na pontuação total do FMS, quando analisada a média dos 77 bombeiros que completaram o estudo.

Por sua vez, Kiesel, Plisky e Butler (2011) testaram a efetividade de um treinamento para melhorar a pontuação do FMS durante as férias de jogadores profissionais adultos de futebol americano, e obtiveram resultados positivos quanto à melhora da pontuação total e diminuição de assimetrias posturais no pós-teste. Tal treinamento foi individualizado com base no resultado do escore total do FMS inicial de cada atleta. Foram sugeridos exercícios "corretivos" de movimento, alongamentos e automassagem para liberação de pontos gatilhos. Os autores também sugeriram que, estatisticamente, o indicativo mais forte de um baixo escore no FMS é a nota baixa no agachamento.

Do mesmo modo, Frost et al. (2012) também utilizaram o FMS para avaliar a efetividade de um treinamento realizado com bombeiros, com duração de uma hora e meia, realizado três vezes por semana ao longo de 12 semanas. O treinamento consistiu em exercícios de mobilidade e controle neuromuscular (de acordo com os resultados individuais do FMS). Os voluntários foram divididos em três grupos (intervenção 1, intervenção 2 e controle) sendo que a diferença entre os grupos "intervenção" foi a ênfase dada na qualidade do movimento durante o treinamento. Cada grupo "intervenção" foi instruído por um profissional especializado em treinamento de força e condicionamento físico (um supervisor por grupo). Os autores não encontraram diferença significante para nenhum dos grupos no pós-treino. Porém, a pontuação de $85 \%$ dos voluntários do grupo controle foi alterada, demonstrando acréscimo ou decréscimo da pontuação em pelo menos um dos movimentos. Os achados do grupo controle do estudo de Frost et al. pode ser explicados pelo estudo de Sprague, Mokha e Gatens (2014). Os autores realizaram acompanhamento de atletas colegiais de futebol e vôlei, apenas documentando a mudança no escore total do FMS durante uma temporada competitiva. Os resultados demonstraram um no DS e no ILL para todos os atletas e ASLR e RS piorado em todos os sujeitos. Também se notou uma redução do número de assimetrias e de escores 1 , mostrando variações da qualidade do movimento de atletas ao longo de uma temporada. 
O FMS também já foi aplicado em crianças. Um exemplo é o estudo produzido por Duncan e Stanley (2012), os quais demonstraram que o peso e no nível de atividade física foram bons preditores da qualidade do movimento funcional nessa população. Mitchell, Johnson e Adamson (2015) realizaram estudo com crianças de 9 a 11 anos, e encontraram uma correlação do escore total do FMS com a força abdominal, mostrando que o teste pode ser usado como uma ferramenta para treinamento de crianças.

Por sua vez, Song et al. (2014) realizaram uma intervenção em atletas escolares de baseball, melhorando, ao final de 16 semanas, a força e a flexibilidade dos atletas. Os treinamentos, personalizados, basearam-se nas pontuações $\leq 2$ de cada movimento do FMS e de cada atleta.

\subsection{FMS no contexto do equilíbrio e propriocepção}

O equilíbrio do ser humano é constituído por uma sucessão ascendente de equilíbrios instáveis controlados pela musculatura tônica. Como todos os corpos em equilíbrio, o corpo humano é submetido às leis da gravidade. Para que esteja em equilíbrio, seu centro de gravidade deve cair no meio da base de sustentação (equilíbrio estável). Se o centro de gravidade "cair" para o lado, para frente ou trás da base de sustentação, caracteriza-se o equilíbrio instável. A estática do ser humano é condicionada pela base de sustentação e pela posição do centro de gravidade. Essa necessidade absoluta leva à grande lei da estática humana: a lei das compensações. De acordo com as possibilidades anatômicas, a compensação poderá ocorrer sobre um ou vários segmentos, sobre uma ou mais articulações, em um ou vários planos (Bienfait 1995).

Assim, a postura pode ser definida pelo alinhamento mecânico de diferentes articulações e segmentos, e pela orientação em relação ao ambiente (ShumwayCook and Woollacott 2003). Nesse sentido, o controle postural envolve o controle da posição do corpo no espaço, com o objetivo de estabilidade e orientação. equilíbrio de um indivíduo envolve a capacidade de manter o seu centro de massa projetado dentro dos limites da base de apoio, determinando os limites de estabilidade corporal (Shumway-Cook and Woollacott 2003). Vale ressaltar que o 
controle postural pode ser influenciado tanto por fatores intrínsecos, aqueles relacionados às características do organismo, como idade, peso corporal, força muscular; quanto aos fatores extrínsecos, ou seja, fatores ambientais, como a superfície de apoio e a postura do individuo.

Uma forma de monitorar o desempenho relacionado ao equilíbrio dinâmico quantitativamente é por meio de plataformas de equilíbrio. As plataformas são dispositivos capazes de mensurar objetivamente e registrar a capacidade do indivíduo de estabilizar a articulação envolvida sob estresse dinâmico. Diferentemente da plataforma de força, as plataformas de equilíbrio, como o Balance System (BIODEX 2011), utiliza uma plataforma circular que pode variar livremente através dos eixos ântero-posterior e medio-lateral, simultaneamente. A confiabilidade do sistema foi demonstrada por estudos realizados em jovens, adultos e idosos fisicamente ativos (Hinman 2000; Cachupe 2001).

Nas últimas décadas, a pesquisa sobre o controle postural e o impacto das suas disfunções tem evoluído, e déficits no equilíbrio estático e dinâmico foram associados com a predição de lesões musculoesqueléticas em indivíduos praticantes de exercício e esportistas (Clifton, Harrison et al. 2013; Teyhen, Bergeron et al. 2014). A mensuração do equilíbrio postural, por sua vez, é comumente utilizada para avaliar o controle postural e função neuromuscular (Clifton, Harrison et al. 2013). Nesse contexto, ter o equilíbrio preservado é essencial para a realização das posturas do FMS (Clifton, Harrison et al. 2013), o qual requer ajustes constantes que são proporcionados pela ação muscular (Alonso, Brech et al. 2011).

Sendo assim, é possível supor que um déficit de equilíbrio postural possa determinar piores desempenhos na execução das posturas propostas pelo FMS, indicando um importante fator de risco para lesões. Além disso, levanta-se uma questão relativa ao peso de diferentes construtos/variáveis no escore final do método (Kazman, Galecki et al. 2013; Li, Wang et al. 2015), e destaca-se que há evidências escassas da associação do FMS com outras medidas físicas de desempenho, como o equilíbrio postural (Teyhen, Shaffer et al. 2014).

Em estudo norte americano de 2013 , que teve por objetivo avaliar a relação entre a pontuação do FMS com mudanças no equilíbrio estático antes e após um programa de exercícios, não foram encontrados resultados positivos. Portanto o 
FMS pode não ser útil para prever quem terá um maior déficit no equilíbrio após o exercício. A avaliação pré e pós-intervenção no mesmo estudo não apontou alteração no escore total do FMS. Entretanto, profissionais de saúde que tenham por objetivo identificar déficits de controle postural podem, de acordo com Clifton et al. (2013), utilizar as posturas HS, o ILL e o ASLR, pois as mesmas apresentam uma relação com o equilíbrio postural (Clifton, Harrison et al. 2013). Por sua vez, Hartigan et al. (2014) realizou estudo com adultos saudáveis fisicamente ativos, tentando relacionar a postura ILL com o equilíbrio, potência e velocidade, e não encontraram relação estatisticamente significativa para nenhuma variável.

Com a disseminação do FMS como método confiável (Minick, Kiesel et al. 2010; Gribble, Brigle et al. 2013), outras formas de sua utilização foram criadas. Boyle, Butler e Queen (2015) testaram o equilíbrio dinâmico e o movimento funcional de adolescentes, 9 meses após reconstrução primária de LCA. Concluiu-se que não haviam recuperado completamente a capacidade funcional a ponto de poderem voltar ao esporte, indicando uma nova forma de controlar a "alta clínica" para os terapeutas, por meio do FMS. De fato, vários autores apresentam resultados mostrando que o equilíbrio dinâmico tem relação com o desempenho funcional, ao realizarem estudos com atletas e chegarem a resultados de diminuição no número de lesões após um treinamento neuromuscular (Paterno, Myer et al. 2004; Plisky, Rauh et al. 2006; Filipa, Byrnes et al. 2010; Steffen, Emery et al. 2013). 


\section{MATERIAIS E MÉTODOS}

\subsection{Desenho do estudo}

Trata-se de um estudo transversal, caracterizado pela aplicação do método FMS em indivíduos jovens e sadios. Os voluntários foram submetidos a testes de equilíbrio e questionários de nível de atividade física e qualidade de vida. As coletas de dados foram realizadas no Laboratório de Análise do Movimento Humano e Processamento de Sinais da Faculdade de Ceilândia, Universidade de Brasília (UnB).

\subsection{Participantes}

Foi realizado um cálculo amostral considerando o poder estatístico de $80 \%$ e um valor de alfa de $5 \%$ (erro tipo I), que indicou uma amostra de 65 sujeitos como suficiente para a realização do estudo.

Participaram do presente estudo 58 voluntários, de ambos os sexos, universitários, de 18 a 35 anos e sem histórico de trauma ou lesões que pudessem gerar algum impedimento na realização das atividades do trabalho. A caracterização da amostra está apresentada na Tabela 1.

Os sujeitos foram recrutados por meio de divulgação via rede social e por folhetos fixados nas dependências da universidade, além de contatos verbais em sala de aula, caracterizando uma amostra de conveniência. Os interessados eram encaminhados ao Laboratório para elucidar dúvidas, verificar se preenchiam os critérios de inclusão e marcar as datas para início das coletas.

Para a participação no estudo, os voluntários deveriam se enquadrar nos critérios de inclusão: (1) idade de 18 a 35 anos; (2) estarem aptos a comparecerem no laboratório em dois dias distintos com diferença de, no máximo, uma semana. Foram excluídos os participantes que: (1) possuíssem história de trauma ou qualquer tipo de cirurgia de natureza musculoesquelética em membros inferiores ou coluna; (2) possuíssem quadro diagnosticado de lombalgia, nos últimos seis meses; (3) possuíssem doença cardiovascular diagnosticada; (4) estivessem com dores nos 
membros inferiores e que, de alguma forma, pudesse interferir no resultado dos testes.

Um total de 58 sujeitos foi avaliado para elegibilidade e inclusos no presente estudo. No entanto, três sujeitos excederam o tempo estipulado como limite entre as duas visitas ao laboratório, resultando em uma amostra final de 55 voluntários.

Tabela 1 Caracterização da amostra

\begin{tabular}{lcccc}
\hline & Idade (anos) & Massa $(\mathbf{k g})$ & Altura $(\mathbf{c m})$ & IMC $\mathbf{( k g / \mathbf { m } ^ { 2 } )}$ \\
\cline { 2 - 5 } & Média (DP) & Média (DP) & Média (DP) & Média (DP) \\
Homens $(\mathbf{n}=\mathbf{2 4})$ & $22,63(4,66)$ & $73,38(11,67)$ & $177,04(6,37)$ & $23,38(3,35)$ \\
Mulheres (n = 31) & $20,94(2,46)$ & $55,87(7,06)$ & $163,48(5,59)$ & $20,91(2,56)$ \\
Total $(\mathbf{n}=\mathbf{5 5})$ & $21,67(3,65)$ & $63,51(12,75)$ & $169,40(8,98)$ & $21,83(3,07)$ \\
\hline
\end{tabular}

Todos os participantes que atenderam os critérios de inclusão foram convidados a participar por meio da assinatura do Termo de Consentimento Livre e Esclarecido (Anexo 1), devidamente aprovado pelo Comitê de Ética em Pesquisa da Faculdade de Saúde, Universidade de Brasília (protocolo CAAE n. 31873814.4.0000.0030).

\subsection{Procedimentos de avaliação}

Os participantes foram submetidos a um processo de avaliação em dois momentos distintos, no Laboratório de Análise do Movimento Humano e Processamento de Sinais. Todas as avaliações ocorreram no turno matutino e em ambiente climatizado.

Em um primeiro momento, cada voluntário respondeu um questionário avaliativo, no qual se coletava o nome completo, data de nascimento, idade, sexo, dominância de pé e mão, massa corporal, altura; uma anamnese, com o propósito 
de checar se o voluntário tinha histórico de traumas, fraturas e lombalgia nos últimos seis meses, assim como antecedentes cirúrgicos e doenças cardiopulmonares; também se indagava sobre os hábitos de vida, como tabagismo e etilismo. Em seguida, aplicaram-se dois questionários: O IPAQ (International Physical Activity Questionnaire) sobre atividade física e o questionário SF-36 de qualidade de vida.

No segundo encontro, o equilíbrio postural foi avaliado na plataforma de equilíbrio. As avaliações sempre começavam com a perna direita e depois com a perna esquerda. Após a avaliação com a plataforma de equilíbrio, um examinador certificado no método FMS avaliava o(a) voluntário(a). Na avaliação do FMS foram utilizadas as sete posições de acordo com protocolo do método.

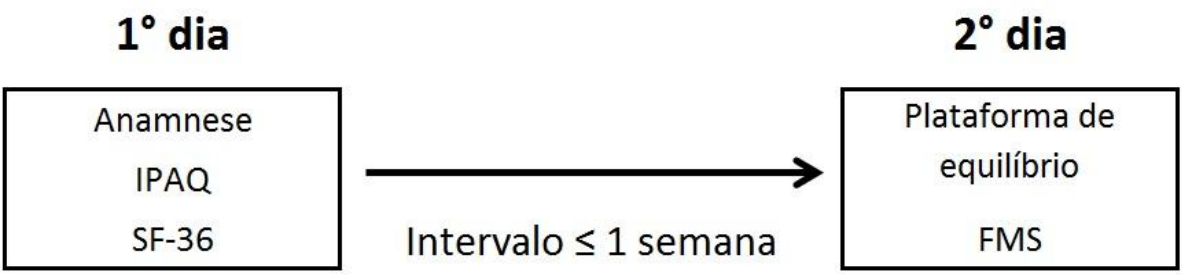

Figura 2. Ilustração que representa o desenho do estudo.

\subsection{Plataforma de equilíbrio}

Para o presente estudo, foi utilizada uma plataforma de equilíbrio Balance System (Biodex Medical Systems, Shirley, New York, USA). A calibração foi realizada de acordo com as especificações do manual do fabricante. A plataforma consiste em uma base circular com mobilidade de até $20^{\circ}$ de inclinação da superfície em uma gama de movimentos em $360^{\circ}$. A plataforma é livre para se mover nos eixos AP (anteroposterior) e ML (médio-lateral) simultaneamente, permitindo a avaliação do equilíbrio postural em condições estáticas e dinâmicas dos membros inferiores.

O protocolo de avaliação adotado foi o teste Athlete Single Leg Stability Testing (ASL) (BIODEX 2011). O ASL foi realizado em duas condições: estável e instável e caracterizado por 2 séries de 20 segundos para ambos os membros inferiores (dominante e não dominante), com 5 minutos de intervalo entre cada 
membro e entre cada condição. Para a condição dinâmica, a plataforma de equilíbrio proporciona níveis de instabilidade variando de 1 a 8, sendo que o aumento do nível impõem maior desafio e instabilidade. Com base em estudo piloto, verificou-se que o nível 4 apresentou desafio e instabilidade suficientes, e que ainda permitisse que os participantes conseguissem finalizar o protocolo de avaliação. Deste modo, o nível 4 foi adotado para a análise da condição instável de equilíbrio no presente estudo.

Os voluntários foram orientados a adotar uma posição com a perna de apoio semi-flexionada e a contralateral com o joelho flexionado a $90^{\circ}$, com braços cruzados e mãos apoiadas nos ombros, baseado no estudo de Paterno et al. (2004).

Com base no teste ASL, foram obtidos os índices: 1) Antero-posterior (AP): Representa a variação do deslocamento da plataforma, no plano sagital; 2) Mediolateral (ML): Representa a variação do deslocamento da plataforma, no plano frontal; e 3) Equilíbrio global (EG): representação da variação global do deslocamento da plataforma. Para todos os índices, altos valores representam uma quantidade elevada de movimento na plataforma e, por conseguinte, indicam possíveis distúrbios de equilíbrio (Akhbari, Ebrahimi Takamjani et al. 2007; Pereira, de Campos et al. 2008; Ku, Abu Osman et al. 2012; Ku, Abu Osman et al. 2012; Corzo, Hernández-Mocholí et al. 2013; Sherafat, Salavati et al. 2014).

\subsection{Questionário internacional de atividade física (IPAQ)}

Existem dois modelos de questionários IPAQ, versão longa e curta (IPAQ-S). A atividade física auto relatada deste estudo foi coletada utilizando o IPAQ-S, por ser de mais fácil realização por parte dos participantes. O IPAQ questiona os participantes quanto a realização de atividades físicas de ao menos 10 minutos ao longo dos últimos 7 dias. A pessoa que está respondendo é instigada a reportar o tempo despendido em atividade física realizada no seu tempo livre, no trabalho, em atividades domésticas, e transporte em três intensidades: caminhada, moderada e vigorosa. Exemplos de atividades que representam cada intensidade são expostos como guia; por exemplo, quando a pessoa é questionada sobre atividades vigorosas assim como "correr, fazer ginástica aeróbica, jogar futebol, pedalar rápido na bicicleta, jogar basquete, fazer serviços domésticos pesados em casa, no quintal ou 
cavoucar no jardim, carregar pesos elevados ou qualquer atividade que fez aumentar muito sua respiração ou batimentos do coração.

A pontuação é realizada de acordo com a frequência e tipo de atividade física realizada, sendo classificada em sedentário, irregularmente ativo, ativo e muito ativo. Os critérios para determinar a classificação de cada sujeito são os seguintes: MUITO ATIVO: aquele que cumpriu as recomendações de:

a) VIGOROSA: $\geq 5$ dias/sem e $\geq 30$ minutos por sessão

b) VIGOROSA: $\geq 3$ dias/sem e $\geq 20$ minutos por sessão + MODERADA e/ou CAMINHADA: $\geq 5$ dias/sem e $\geq 30$ minutos por sessão.

ATIVO: aquele que cumpriu as recomendações de:

a) VIGOROSA: $\geq 3$ dias/sem e $\geq 20$ minutos por sessão; ou

b) MODERADA ou CAMINHADA: $\geq 5$ dias/sem e $\geq 30$ minutos por sessão; ou

c) Qualquer atividade somada: $\geq 5$ dias/sem e $\geq 150$ minutos/sem (caminhada + moderada + vigorosa)

IRREGULARMENTE ATIVO: aquele que realiza atividade física porém insuficiente para ser classificado como ativo pois não cumpre as recomendações quanto à freqüência ou duração.

SEDENTÁRIO: aquele que não realizou nenhuma atividade física por pelo menos 10 minutos contínuos durante a semana.

\subsection{Questionário de qualidade de vida (SF-36)}

O SF-36 (Medical Outcomes Stydy 36 - Item Short-Form Health Survey) é um questionário de qualidade de vida, de fácil administração e compreensão, bem conhecido e validado (Brazier, Harper et al. 1992; Fukuhara, Bito et al. 1998), que gera um perfil do paciente baseado em oito escalas com 36 questões auto- 
administradas: capacidade funcional, aspectos físicos, dor, estado geral da saúde, vitalidade, aspectos sociais, aspectos emocionais e saúde mental. O número de perguntas para cada domínio varia de 2 a 10. Os escores variam de 0 (estado de saúde ruim) a 100 (melhor estado de saúde). O SF-36 é uma ferramenta útil para acompanhamento da evolução da saúde na população em geral (Hemingway, Stafford et al. 1997).

\subsection{Functional Movement Screen (FMS)}

O FMS é composto por sete posturas (Figura 1): Deep Squat (agachamento profundo que avalia bilateralmente e simetricamente, a funcionalidade dos quadris, joelhos e tornozelos), Hurdle Step (um passo através de um obstáculo, examinando a mecânica da passada), In line Lunge (mobilidade do quadril e tronco, estabilidade de tornozelo e joelho, e flexibilidade dos isquiotibiais), Shoulder Mobility (mobilidade do ombro bilateralmente), Active Straight-Leg Raise (levantar a perna ativamente em extensão de joelho, determinando a atividade do tendão e flexibilidade do gastrocnêmio-sóleo, mantendo ao mesmo tempo uma pélvis estável), Trunk Stability Push-up (estabilidade do tronco, enquanto a simetria de membros superiores é executada), Rotary Stability (teste da estabilidade de rotação, que avalia a estabilidade do tronco enquanto os membros superiores e inferiores estão em movimento combinado) (Cook, Burton et al. 2006; Kiesel, Plisky et al. 2007).

A pontuação de cada postura varia de 1 a 3 , sendo a pontuação baseada na qualidade do movimento, presença de assimetrias e dificuldades em completar o teste, de modo que um escore " 3 " indica um movimento ideal e o escore " 1 " um movimento que apresenta déficits de acordo com o esperado para cada postura, baseando-se nas recomendações do método (Cook, Burton et al. 2006).

Após a classificação de cada postura, realiza-se um somatório dos escores individuais. O valor máximo do escore final do FMS é "21", e de acordo com estudos prévios (Cook, Burton et al. 2006; Kiesel, Plisky et al. 2007), escores iguais ou menores que 14 foram associados a um maior risco de lesão, comparados aos que apresentam escores mais elevados (>14), indicando que o ponto de corte "14" é sensível à detecção e predição de lesões musculoesqueléticas e determinação do 
nível de funcionalidade e qualidade de movimento pelo FMS. Deste modo, para o presente estudo, o ponto de corte com escore "14" no escore final do FMS foi utilizado como critério para divisão dos grupos: FMS $_{1}$ (indivíduos classificados com escore >14) e $\mathrm{FMS}_{2}$ (indivíduos classificados com escore $\leq 14$ ).

\subsection{Análise dos dados}

Para a análise dos dados foi utilizado o programa SPSS (Statistical Package for Social Sciences) versão 22.0. A variável independente foi o grupo classificado pelo $F M S\left(F M S_{1}\right.$ e $\left.F M S_{2}\right)$, e as variáveis dependentes foram os índices de equilíbrio postural (antero-posterior - AP; médio-lateral - ML e equilíbrio global - EG); domínios de qualidade de vida (capacidade funcional, aspectos físicos, dor, saúde geral, vitalidade, social, emocional e saúde mental); índice de massa corporal e classificação do IPAQ.

Inicialmente, aplicou-se o teste de Shapiro-Wilk com o intuito de verificar a normalidade dos dados. Para os índices de equilíbrio, IMC, domínios de qualidade de vida (saúde geral e vitalidade), os pressupostos foram atendidos e os dados são apresentados pela média (desvio-padrão). Para as demais variáveis (capacidade funcional, aspectos físicos, dor, social, emocional e saúde mental), como os pressupostos não foram atendidos, os dados são apresentados pela mediana e quartis $(25 \%$ e $75 \%)$.

Inicialmente, utilizou-se o teste t de Student para amostras pareadas com o intuito de comparar os índices de equilíbrio entre os membros inferiores dominantes e não dominante. Como não foram encontradas diferenças significantes, em nenhuma das medidas de equilíbrio (EG, AP e ML) nas condições estável e instável, os dados apresentados referem-se às medidas do membro dominante. O teste $t$ de Student para amostras independentes foi utilizado para verificar diferenças entre os grupos $\mathrm{FMS}_{1}$ e $\mathrm{FMS}_{2}$ em relação às variáveis dependentes $\mathrm{EG}, \mathrm{ML}$ e AP.

$O$ teste de Mann-Whitney foi aplicado para se verificar diferenças entre os grupos $\mathrm{FMS}_{1}$ e $\mathrm{FMS}_{2}$ em relação às variáveis dependentes não paramétricas (domínios de qualidade de vida: capacidade funcional, aspectos físicos, dor, social, 
emocional e saúde mental). Com o intuito de se verificar a associação entre o nível de atividade física e o grupamento $\mathrm{FMS}_{1}$ e $\mathrm{FMS}_{2}$, aplicou-se o teste de Quiquadrado.

Aplicou-se uma análise discriminante, com o intuito de identificar quais das variáveis melhor discriminariam ou distinguiriam os dois grupos de classificação do FMS (FMS 1 e $F M S_{2}$ ). Para a análise discriminante, a homogeneidade das matrizes de variância-covariância foi testada pelo teste Box's M. Três análises discriminantes foram realizadas pelo método Wilk's Lambda para identificar (1) quais das medidas de equilíbrio ( $A P, M L$ e $E G$ ), em condições estáticas e dinâmicas, discriminariam significativamente os grupos $\mathrm{FMS}_{1}$ e $\mathrm{FMS}_{2}$; (2) Se o IMC discrimina os grupos FMS e $\mathrm{FMS}_{2}$ e, por fim (3) Se os domínios de qualidade de vida discriminariam significativamente os grupos $\mathrm{FMS}_{1}$ e $\mathrm{FMS}_{2}$. A correlação canônica foi usada para medir a associação entre os escores discriminantes e o grupo de indivíduos classificados pelo FMS. Posteriormente, a análise classificatória foi aplicada para demonstrar a precisão de alocação dos participantes nos grupos, sendo confirmado por uma validação cruzada. A significância adotada foi de $5 \%(p<0,05)$. 


\section{RESULTADOS}

\subsection{Equilíbrio postural}

Os dados referentes aos índices de equilíbrio global (EG), anteroposterior (AP) e médio-lateral (ML), nas condições estável e instável da plataforma de equilíbrio estão apresentados nas Figuras 3, 4 e 5, respectivamente, em ambos os grupos analisados $\left(\mathrm{FMS}_{1}\right.$ e $\left.F \mathrm{FS}_{2}\right)$.

Quando os grupos $\mathrm{FMS}_{1}$ e $\mathrm{FMS}_{2}$ foram comparados nas condições estável e instável, verificou-se uma diferença significante no índice AP na condição instável, indicando maiores valores para o grupo $\mathrm{FMS}_{2}(p=0,017)$. Não foram encontradas diferenças significantes para as demais comparações $(p>0,05)$.

Em relação aos índices de equilíbrio em condições estável, verificou-se que nenhuma das variáveis discriminou os grupos de sujeitos do $\mathrm{FMS}_{1}$ e $\mathrm{FMS}_{2}(\lambda=0,92$; $\left.X^{2}=4,17 ; p=0,24\right)$. Do mesmo modo, os índices de equilíbrio na condição instável também não discriminaram os sujeitos do $\mathrm{FMS}_{1}$ e $\mathrm{FMS}_{2}\left(\lambda=0,86 ; \mathrm{X}^{2}=7,33 ; p=0,06\right)$. 


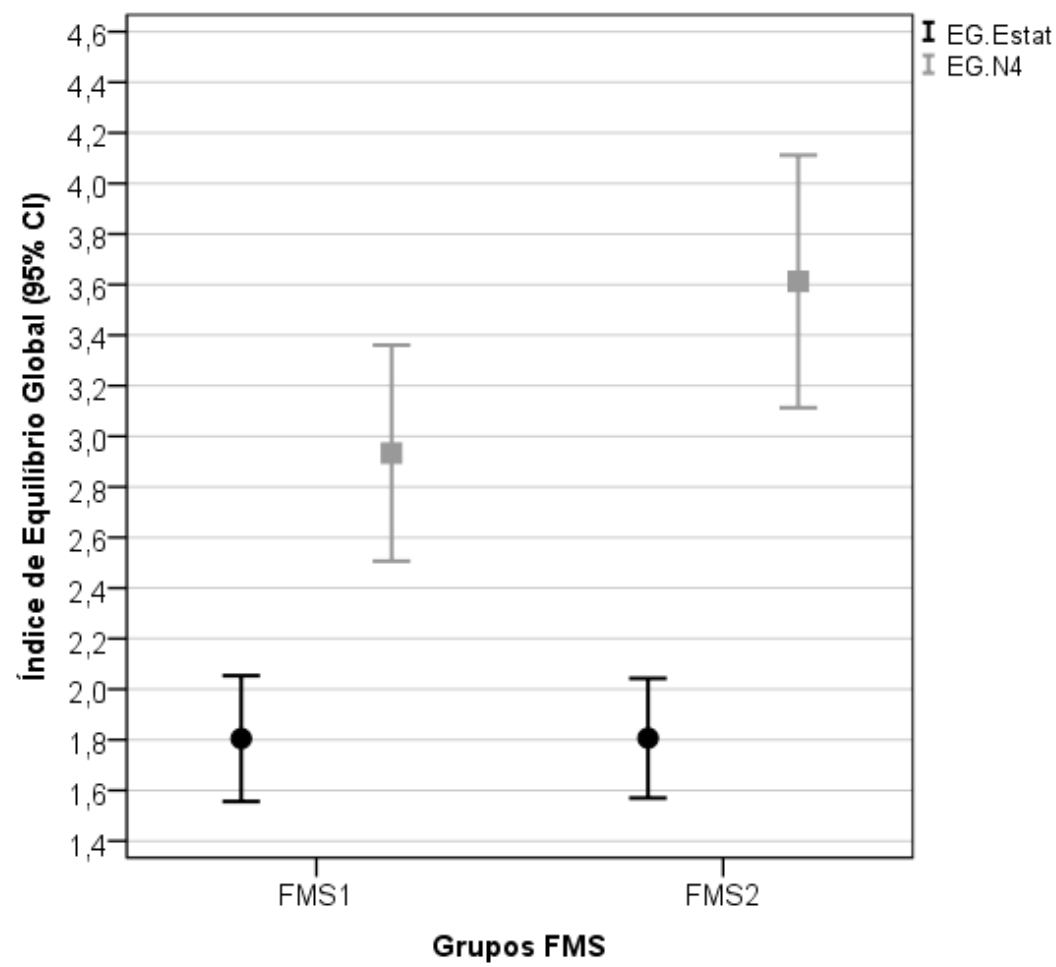

Figura 3. Índice de Equilíbrio Global (EG) nas condições estáveis (Estat) e instáveis (N4), entre os grupos $\mathrm{FMS}_{1}$ (escore $>14$ ) e $\mathrm{FMS}_{2}$ (escore $\leq 14$ ).

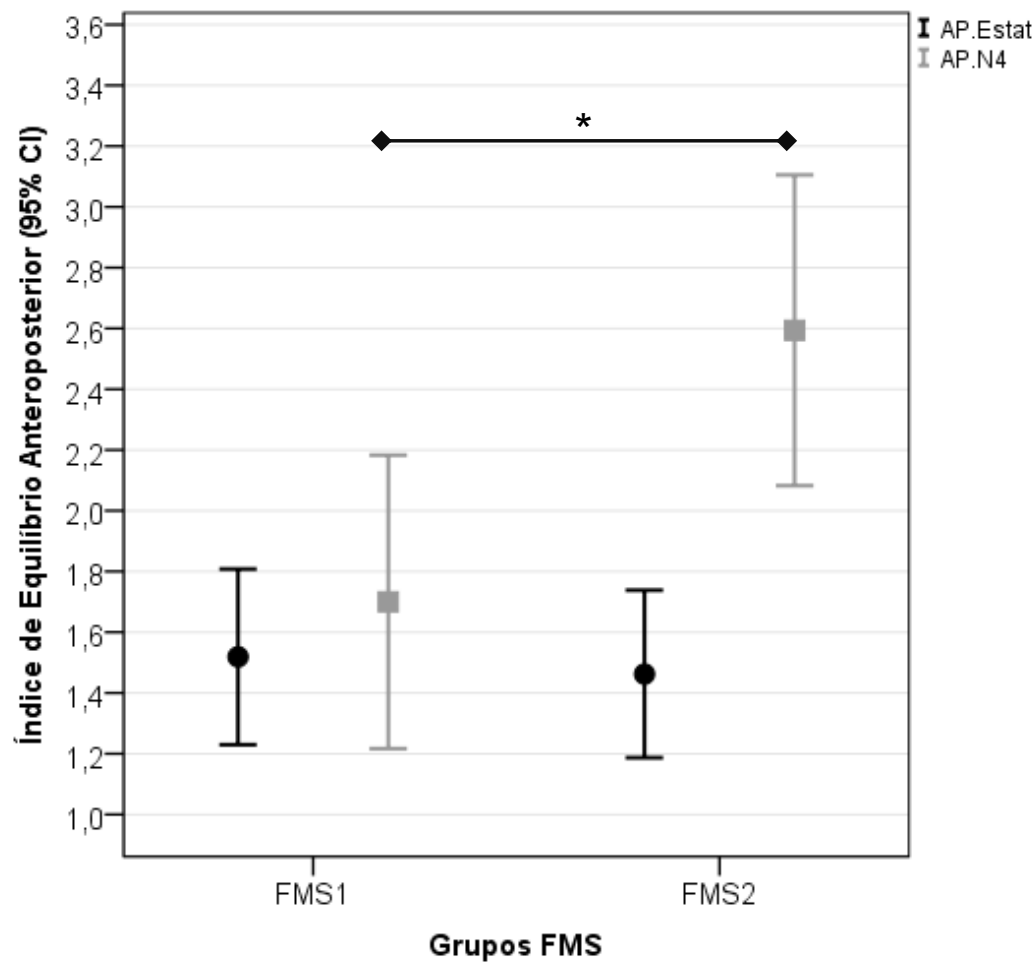

Figura 4. Índice de Equilíbrio Anteroposterior (AP nas condições estáveis (Estat) e instáveis (N4), entre os grupos $\mathrm{FMS}_{1}$ (escore >14) e $\mathrm{FMS}_{2}$ (escore $\leq 14$ ) (*diferença significante entre os grupos: $p=0,017)$. 


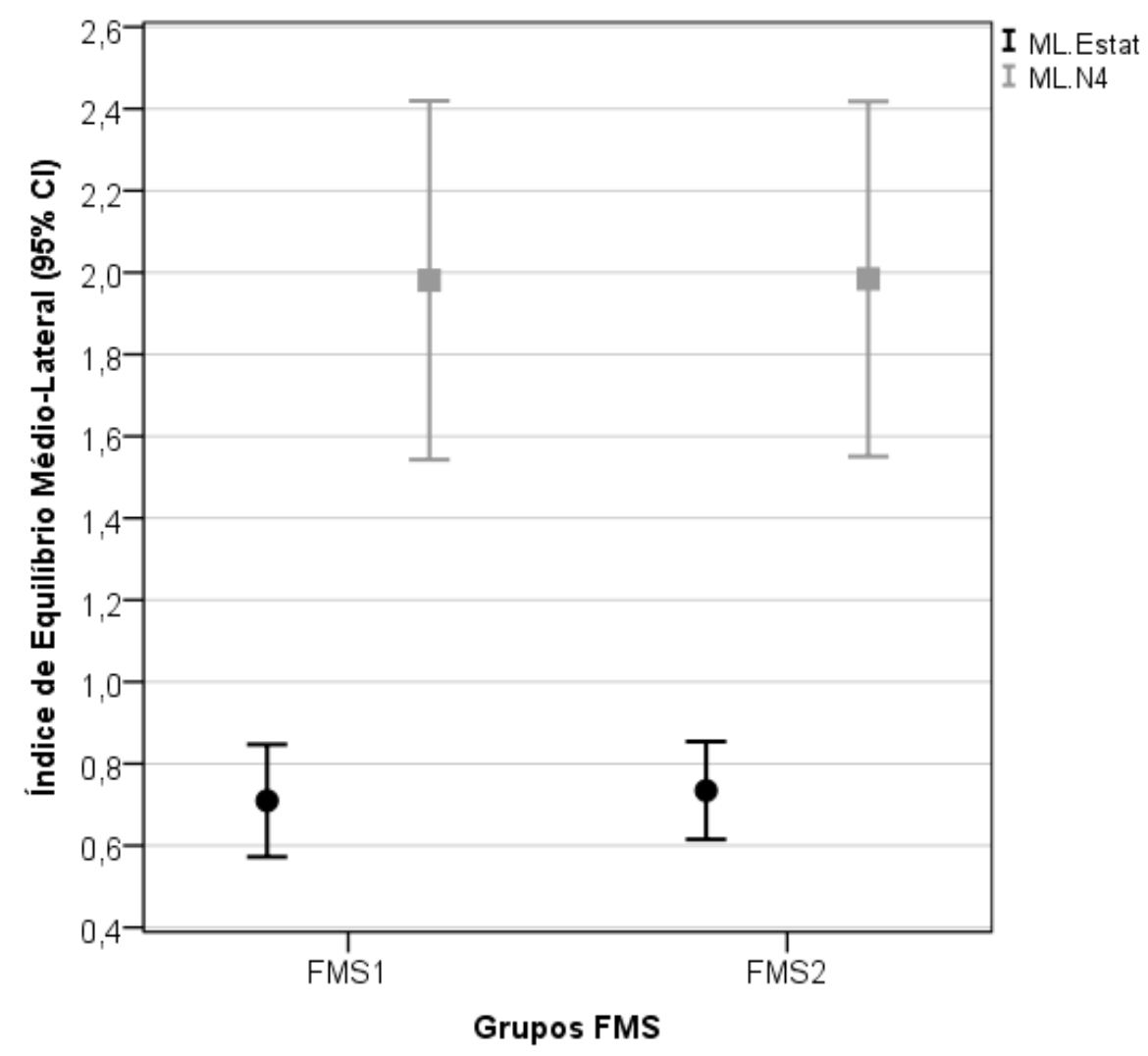

Figura 5. Índice de Equilíbrio Médio-Lateral (ML) nas condições estáveis (Estat) e instáveis (N4), entre os grupos $\mathrm{FMS}_{1}$ (escore >14) e FMS 2 (escore $\leq 14$ ).

\subsection{IMC}

O IMC como função discriminante dos grupos FMS1 e FMS2, não foi significativo. A variável teve baixo valor na correlação canônica (.004) o que quer dizer que a proporção do total de variância explicado pelo IMC é muito baixo, ou seja, baixo grau de associação entre a função discriminante e os grupos. Além disso, houve um decréscimo no poder discriminatório por conta do aumento no valor do lambda de Wilks (.976) $\left(\lambda=0,97 ; X^{2}=0,44 ; p=0,80\right)$. 


\subsection{Qualidade de Vida}

Os resultados referentes aos domínios de qualidade de vida estão apresentados na Tabela 2, onde os dados estão divididos de acordo com os níveis de classificação do FMS e na forma de média e desvio padrão para "saúde geral" e "vitalidade", mediana e quartis para os demais domínios.

Tabela 2. Domínios de qualidade de vida dos participantes do estudo, com base no questionário SF-36.

\begin{tabular}{|c|c|c|c|}
\hline & Grupos & Média & DP \\
\hline \multirow{2}{*}{ Saúde Geral } & $\mathrm{FMS}_{1}$ & 70,60 & 2,99 \\
\hline & $\mathrm{FMS}_{2}$ & 79,72 & 3,49 \\
\hline \multirow{3}{*}{ Vitalidade } & $\mathrm{FMS}_{1}$ & 55,28 & 2,68 \\
\hline & $\mathrm{FMS}_{2}$ & 65,00 & 3,22 \\
\hline & Grupo FMS & Mediana & Quartis (25-75\%) \\
\hline \multirow{2}{*}{ Capacidade Funcional } & $\mathrm{FMS}_{1}$ & 95,00 & $85,00-100,00$ \\
\hline & $\mathrm{FMS}_{2}$ & 90,00 & $85,00-100,00$ \\
\hline \multirow{2}{*}{ Aspectos Físicos } & $\mathrm{FMS}_{1}$ & 87,50 & $75,00-100,00$ \\
\hline & $\mathrm{FMS}_{2}$ & 100,00 & $75,00-100,00$ \\
\hline \multirow{2}{*}{ Dor } & $\mathrm{FMS}_{1}$ & 74,00 & $64,50-100,00$ \\
\hline & $\mathrm{FMS}_{2}$ & 72,00 & $51,00-84,00$ \\
\hline \multirow{2}{*}{ Aspectos Sociais } & $\mathrm{FMS}_{1}$ & 87,50 & $75,00-100,00$ \\
\hline & $\mathrm{FMS}_{2}$ & 100,00 & $75,00-100,00$ \\
\hline \multirow{2}{*}{ Aspectos Emocionais } & $\mathrm{FMS}_{1}$ & 100,00 & $33,30-100,00$ \\
\hline & $\mathrm{FMS}_{2}$ & 100,00 & $33,30-100,00$ \\
\hline \multirow{2}{*}{ Saúde Mental } & $\mathrm{FMS}_{1}$ & 80,00 & $68,00-88,00$ \\
\hline & $\mathrm{FMS}_{2}$ & 80,00 & $64,00-84,00$ \\
\hline
\end{tabular}

DP: Desvio-padrão;

FMS1: escore $\leq 14$; FMS2: escore $>14$ 
Não foram encontradas diferenças entre os grupos $\mathrm{FMS}_{1}$ e $\mathrm{FMS}_{2}$ em relação à capacidade funcional, aspectos físicos, dor, aspectos sociais, emocionais e saúde mental.

A análise discriminante para os domínios de qualidade de vida não foi significante nas funções discriminantes, para todas as variáveis. Correlação canônica (.474) e lambda de Wilks (.132). Foi possível verificar que houve um baixo grau de associação entre a função discriminante e os grupos, com baixo poder discriminatório.

\subsection{Nível de Atividade Física}

Os achados referentes à associação entre o nível de atividade física e a classificação do FMS estão apresentados na Tabela 3.

Os achados demonstraram que não houve associação significante entre a condição física classificada pelo IPAQ e o grupo no qual o indivíduo foi alocado $\left(\mathrm{FMS}_{1}\right.$ ou $\left.\mathrm{FMS}_{2}-\mathrm{X}^{2}=0.69 ; p>0,05\right)$

Tabela 3. Nível de atividade física e classificação do FMS.

\begin{tabular}{cc|c|c|c|c|c}
\hline \multirow{2}{*}{} & \multicolumn{4}{|c|}{ Questionário de Atividade Física } & \multirow{2}{*}{ Total } \\
\cline { 2 - 6 } & Sedentário & $\begin{array}{c}\text { Irregularmente } \\
\text { Ativo A }\end{array}$ & Ativo & $\begin{array}{c}\text { Muito } \\
\text { Ativo }\end{array}$ & \\
\hline \multirow{2}{*}{ Grupo FMS } & $<=14$ & 2 & 13 & 12 & 8 & 35 \\
\cline { 2 - 6 } & $>14$ & 0 & 9 & 6 & 5 & 20 \\
\hline \multicolumn{2}{|c|}{ Total } & 2 & 22 & 18 & 13 & 55 \\
\hline
\end{tabular}




\section{DISCUSSÃO}

\subsection{Equilíbrio Postural}

Os achados do presente estudo demonstraram maiores valores quanto ao índice AP instável para os indivíduos alocados no grupo $\mathrm{FMS}_{2}$, diferindo do esperado baseado no estudo de Paterno et al. (2004), onde uma variação ML seria o de uma coerência maior. Paterno et al. (2004) demonstraram relação próxima entre a estabilidade total e a anteroposterior, mas não entre estabilidade total e médiolateral. Além disso, os autores levantaram a hipótese de que o déficit no plano coronal é um fator de risco para lesão de LCA, o que reafirma a necessidade de avaliação nos diferentes planos (AP, ML e EG).

Clifton et al. (2013) analisaram a relação do equilíbrio estático pós exercício com o resultado do FMS e não encontrou relação. Mesmo não sendo o foco do deste estudo, é importante comentar este pelo fato de lesões de tornozelo serem comuns em populações ativas fisicamente, resultando em possíveis instabilidades crônicas, degeneração articular e comoção negativa na qualidade de vida (Lohmander, 2004). Tais lesões ocorrem mais comumente ao final da prática de exercícios prolongados e/ou intensos (Fernandez, 2007). No referido estudo, o FMS não diferiu antes e após a realização de exercício.

Os resultados encontrados corroboram com os achados de Lockie et al. (2015) que identificou limitada capacidade do FMS em identificar equilíbrio dinâmico em atletas. Independente da classificação em que os voluntários se encontravam no estudo (alta, intermediária ou baixa performance no teste de equilíbrio).

Os resultados apresentados nesse estudo dão suporte aos achados anteriores que mostraram relação limitada ou nenhuma entre o escore total do FMS e performance atlética. (Okada, Huxel et al. 2011; Parchmann and McBride 2011; Lockie, Schultz et al. 2015). 


\subsection{IMC}

Os achados com relação ao IMC, demonstraram que esta variável não discrimina os valores de pontuação do FMS, contrariamente ao estudo de Duncan e Stanley (2012). Duncan et al. (2013) demonstraram a existência de uma relação indiretamente proporcional quanto ao IMC e FMS, indicando que quanto maior o IMC dos voluntários, menor era a pontuação total do FMS. Essa divergência pode ser explicada pelas diferenças entre os estudos. Primeiro, a amostra de Duncan foi composta por crianças (10 a 11 anos), diferentemente cuja composição foi por voluntários de 18 à 35 anos; houve assim diferença na média de pontuação FMS total entre sua amostra e a do presente estudo (14,9 e13,29 respectivamente); e na média de IMC para ambos os sexos, onde nosso resultado médio foi de 21,99 e o estudo de Duncan obteve pontuação média de 14. Apesar dessa diferença na média da pontuação do IMC dos sujeitos entre os estudos, nosso estudo não incluiu indivíduos acondicionados na categoria de "sobrepeso" do IMC. Por outro lado, o estudo de Duncan teve $33 \%$ de sua amostra nessa categoria. Julgamos que tal diferença possa ser o diferencial do achado de Duncan quanto à relação entre IMC e FMS total. Também é possível supor que a ausência de voluntários em nosso estudo classificados em diferentes categorias do IMC possa não ter dado profundidade suficiente par ao IMC influenciar um resultado significativo.

Tal hipótese também é defendida por Mitchell et al. (2015), que observaram também a não relação do IMC com o movimento funcional, corroborando assim com nossos achados. O autor ainda sugeriu que variáveis como flexibilidade e força possam ter maior relação com a pontuação do FMS.

Lloyd (2015), cujo estudo demonstrou que o nível de maturação dos jovens tem papel decisivo no desempenho funcional dos mesmos, executou em seu estudo com jovens jogadores de futebol entre 11 e 16 anos. Todos realizaram testes de maturação, FMS e atividades de performance física, onde os jogadores mais velhos tiveram desempenho significativamente melhor para todos os testes de performance. Este dado passa a ser importante ao relacionarmos uma avaliação estrutural (IMC) com uma funcional (FMS). A estrutura corporal pode, portanto, ter atribuição na diferença de resultados funcionais dos artigos discutidos. 


\subsection{Qualidade de Vida e Nível de Atividade Física}

Nossos resultados demonstraram que os grupos do FMS não foram discriminados por nenhum dos domínios de qualidade de vida. Este achado pode ser explicado pelo fato de que os autores do FMS afirmar que a função do método é a prevenção de lesão e performance atlética. Tal prevenção ocorre por meio de recomendações funcionais em protocolos de capacidade física. Ainda, vale salientar que o mesmo foi criado a partir de princípios fundamentais de cinesiologia e propriocepção, fatores estruturalmente físicos (Cook, Burton et al. 2006; Cook, Burton et al. 2006).

Por outro lado, era de se esperar que a qualidade de vida poderia gerar influências, considerando que lesões em atletas são comprovadamente multifatoriais. No entanto, estudos recentes foram realizados com base em fatores individuais. Nesse sentido, o FMS é um método que analisa vários fatores como flexibilidade, equilíbrio e força (McKay, Goldie et al. 2001). Treinamentos de prevenção de lesão normalmente utilizam alguma combinação de fortalecimento, flexibilidade, pliometria e equilíbrio. Além de diminuir a incidência de lesão, pode melhorar a performance atlética (Paterno, Myer et al. 2004).

Contudo, nosso estudo não encontrou associação entre o nível de atividade física com o resultado do FMS. Tais achados vem ao encontro de Duncan e Stanley (2012), os quais constataram uma relação diretamente proporcional entre FMS e atividade física. Os autores demonstraram que quanto maior o nível de atividade física, melhor o resultado total do FMS, o que levou os mesmos a inferirem uma melhor qualidade do movimento funcional desses indivíduos. Porém, o fato do seu estudo ter sido realizado com crianças pode ter sido fator determinante do conflito de resultados, considerando diversos fatores que diferenciam essas populações como maturação corporal e, principalmente, engrama muscular.

Um fato que pode explicar a ausência de associação entre nível de atividade física e o FMS, é o ato de se movimentar, mas sem "qualidade de movimento". É possível supor que uma pessoa que tenha limitações funcionais lance mão de estratégias compensatórias articulares e/ou musculares para tentar desenvolver da 
melhor forma as necessidades corporais ao longo de sua vida. Tais experiências físicas (desportivas ou não), "moldam" a forma como cada indivíduo movimenta-se, não necessariamente sendo da forma mais apropriada. O fato de uma pessoa praticar atividades de forma regular gera benefícios à saúde, conjuntamente com desgastes osteomioarticulares que poderão ser potencializados ou não, de acordo com a sua qualidade de movimento. Tal fato é citado por Foran (2001) em sua pirâmide de desempenho.

O presente estudo possui algumas limitações. Uma delas foi o pequeno número amostral, que pode ter ocasionado um erro tipo II. Outras limitações, como a não padronização da amostra quanto ao esporte praticado, nível de atividade física e/ou composição corporal também podem ser citados.

Nesse caso, sugere-se futuras pesquisas com maior número amostral e continuidade das investigações de outros aspectos, físicos ou não, para tentar discriminar os grupos do FMS, como força muscular e estresse.

\section{CONCLUSÕES}

O presente estudo demonstrou que o nível de atividade física, domínios de qualidade de vida e índices de equilíbrio unipodal de universitários sadios não discriminaram os grupos $\mathrm{FMS}_{1}$ e $\mathrm{FMS}_{2}$.

Tais achados reforçam a importância de se rever o uso do FMS enquanto única estratégia para prevenção de lesões ou análise de desempenho durante atividades físicas e esportivas. 


\section{REFERÊNCIAS}

Akhbari, B., I. Ebrahimi Takamjani, et al. (2007). "A 4-week biodex stability exercise program improved ankle musculature onset, peak latency and balance measures in functionally unstable ankles." Physical Therapy in Sport 8(3): 117-129.

Alonso, A. C., G. C. Brech, et al. (2011). "The influence of lower-limb dominance on postural balance." Sao Paulo Med J 129(6): 410-413.

Bienfait, M. (1995). Fáscias e pompages, Summes Editorial.

BIODEX (2011). Balance System SD. Operation/Service Manual. Shirley/NY, Biodex Medical Systems. 
Bodden, J. G., R. A. Needham, et al. (2013). "The Effect of an Intervention Program on Functional Movement Screen Test Scores in Mixed Martial Arts Athletes." $\underline{J}$ Strength Cond Res.

Boyle, M. J., R. J. Butler, et al. (2015). "Functional Movement Competency and Dynamic Balance After Anterior Cruciate Ligament Reconstruction in Adolescent Patients." J Pediatr Orthop.

Bradley, H. and J. Esformes (2014). "Breathing pattern disorders and functional movement." Int J Sports Phys Ther 9(1): 28-39.

Brazier, J. E., R. Harper, et al. (1992). "Validating the SF-36 health survey questionnaire: new outcome measure for primary care." BMJ 305(6846): 160-164.

Burton, L. (2011). Research Statement and Review.

Butler, R. J., M. Contreras, et al. (2013). "Modifiable risk factors predict injuries in firefighters during training academies." Work.

Cachupe, W. S., B; Kahanov, L; Wughalter, EH (2001). "Reliability of Biodex Balance System Measures." Measurement in Physical Education and Exercise Science 5(2).

Chapman, R. F., A. S. Laymon, et al. (2014). "Functional movement scores and longitudinal performance outcomes in elite track and field athletes." Int J Sports Physiol Perform 9(2): 203-211.

Chorba, R. S., D. J. Chorba, et al. (2010). "Use of a functional movement screening tool to determine injury risk in female collegiate athletes." $\mathrm{N} \mathrm{Am} \mathrm{J} \mathrm{Sports} \mathrm{Phys} \mathrm{Ther}$ 5(2): 47-54.

Clifton, D. R., B. C. Harrison, et al. (2013). "Relationship between functional assessments and exercise-related changes during static balance." J Strength Cond Res 27(4): 966-972.

Cook, G., L. Burton, et al. (2006). "Pre-participation screening: the use of fundamental movements as an assessment of function - part 1." $\mathrm{N} \mathrm{Am} \mathrm{J} \mathrm{Sports} \mathrm{Phys}$ Ther 1(2): 62-72.

Cook, G., L. Burton, et al. (2006). "Pre-participation screening: the use of fundamental movements as an assessment of function - part 2." $\underline{\mathrm{N} \mathrm{Am} \mathrm{J} \mathrm{Sports} \mathrm{Phys}}$ Ther 1(3): 132-139.

Corzo, H., M. A. Hernández-Mocholí, et al. (2013). "Fiabilidad de la prueba Fall Risk de la plataforma Biodex Balance System $\AA$ en las personas mayores institucionalizadas con miedo a caerse mediante test-retest de 12 semanas." Rehabilitación 47(2): 64-70.

Cowen, V. S. (2010). "Functional fitness improvements after a worksite-based yoga initiative." J Bodyw Mov Ther 14(1): 50-54. 
Dossa, K., G. Cashman, et al. (2014). "Can injury in major junior hockey players be predicted by a pre-season functional movement screen - a prospective cohort study." J Can Chiropr Assoc 58(4): 421-427.

Duncan, M. J. and M. Stanley (2012). "Functional movement is negatively associated with weight status and positively associated with physical activity in british primary school children." J Obes 2012: 697563.

Duncan, M. J., M. Stanley, et al. (2013). "The association between functional movement and overweight and obesity in British primary school children." BMC Sports Sci Med Rehabil 5(1): 11.

Filipa, A., R. Byrnes, et al. (2010). "Neuromuscular training improves performance on the star excursion balance test in young female athletes." $\mathrm{J}$ Orthop Sports Phys Ther 40(9): 551-558.

Foran, B. (2001). High-Performance Sports Conditioning.

Frost, D. M., T. A. C. Beach, et al. (2012). "Using the Functional Movement Screen ${ }^{\mathrm{TM}}$ to evaluate the effectiveness of training." J Strength Cond Res 26(6): 1620-1630.

Fukuhara, S., S. Bito, et al. (1998). "Translation, adaptation, and validation of the SF36 Health Survey for use in Japan." J Clin Epidemiol 51(11): 1037-1044.

Garrison, M., R. Westrick, et al. (2015). "Association between the functional movement screen and injury development in college athletes." Int J Sports Phys Ther 10(1): 21-28.

Gribble, P. A., J. Brigle, et al. (2013). "Intrarater reliability of the functional movement screen." J Strength Cond Res 27(4): 978-981.

Hartigan, E. H., M. Lawrence, et al. (2014). "Relationship of the functional movement screen in-line lunge to power, speed, and balance measures." Sports Health 6(3): 197-202.

Hemingway, H., M. Stafford, et al. (1997). "Is the SF-36 a valid measure of change in population health? Results from the Whitehall II study." BMJ 315(7118): 1273-1279.

Hinman, M. R. (2000). "Factors Affecting Reliability of the Biodex Balance System: A Summary of Four Studies." JSR 9(3).

Kazman, J. B., J. Galecki, et al. (2013). "Factor Structure of the Functional Movement Screen in Marine Officer Candidates." J Strength Cond Res.

Kiesel, K., P. Plisky, et al. (2011). "Functional movement test scores improve following a standardized off-season intervention program in professional football players." Scand J Med Sci Sports 21(2): 287-292.

Kiesel, K., P. J. Plisky, et al. (2007). "Can Serious Injury in Professional Football be Predicted by a Preseason Functional Movement Screen?" N Am J Sports Phys Ther 2(3): 147-158. 
Krumrei, K., M. Flanagan, et al. (2014). "The Accuracy of the Functional Movement Screen ${ }^{\mathrm{TM}}$ to Identify Individuals with an Elevated Risk of Musculoskeletal Injury." $\underline{\mathrm{J}}$ Sport Rehabil.

Ku, P. X., N. A. Abu Osman, et al. (2012). "Biomechanical evaluation of the relationship between postural control and body mass index." J Biomech 45(9): 16381642.

Ku, P. X., N. A. Abu Osman, et al. (2012). "The effect on human balance of standing with toe-extension." PLoS One 7(7): e41539.

Letafatkar, A., M. Hadadnezhad, et al. (2014). "Relationship between functional movement screening score and history of injury." Int J Sports Phys Ther 9(1): 21-27.

$\mathrm{Li}, \mathrm{Y}$., X. Wang, et al. (2015). "Exploratory factor analysis of the functional movement screen in elite athletes." J Sports Sci 33(11): 1166-1172.

Lisman, P., F. G. O'Connor, et al. (2013). "Functional movement screen and aerobic fitness predict injuries in military training." Med Sci Sports Exerc 45(4): 636-643.

Lloyd, R. S., J. L. Oliver, et al. (2015). "Relationships between functional movement screen scores, maturation and physical performance in young soccer players." $\underline{J}$ Sports Sci 33(1): 11-19.

Lockie, R., A. Schultz, et al. (2015). "A preliminary investigation into the relationship between functional movement screen scores and athletic physical performance in female team sport athletes." Biol Sport 32(1): 41-51.

McKay, G., P. Goldie, et al. (2001). "Ankle injuries in basketball: injury rate and risk factors." British Journal of Sports Medicine 35(2): 103-108.

Minick, K. I., K. B. Kiesel, et al. (2010). "Interrater reliability of the functional movement screen." J Strength Cond Res 24(2): 479-486.

Mitchell, U. H., A. W. Johnson, et al. (2015). "Relationship between functional movement screen scores, core strength, posture, and body mass index in school children in moldova." J Strength Cond Res 29(5): 1172-1179.

O'Connor, F. G., P. A. Deuster, et al. (2011). "Functional movement screening: predicting injuries in officer candidates." Med Sci Sports Exerc 43(12): 2224-2230.

Okada, T., K. C. Huxel, et al. (2011). "Relationship between core stability, functional movement, and performance." J Strength Cond Res 25(1): 252-261.

Parchmann, C. J. and J. M. McBride (2011). "Relationship between functional movement screen and athletic performance." J Strength Cond Res 25(12): 33783384.

Parenteau-G, E., N. Gaudreault, et al. (2014). "Functional movement screen test: a reliable screening test for young elite ice hockey players." Phys Ther Sport 15(3): 169-175. 
Paterno, M. V., G. D. Myer, et al. (2004). "Neuromuscular training improves singlelimb stability in young female athletes." J Orthop Sports Phys Ther 34(6): 305-316.

Peate, W. F., G. Bates, et al. (2007). "Core strength: a new model for injury prediction and prevention." J Occup Med Toxicol 2: 3.

Pereira, H. M., T. F. de Campos, et al. (2008). "Influence of knee position on the postural stability index registered by the Biodex Stability System." Gait Posture 28(4): 668-672.

Plisky, P. J., M. J. Rauh, et al. (2006). "Star Excursion Balance Test as a predictor of lower extremity injury in high school basketball players." J Orthop Sports Phys Ther 36(12): 911-919.

Sherafat, S., M. Salavati, et al. (2014). "Effect of dual-tasking on dynamic postural control in individuals with and without nonspecific low back pain." $\mathrm{J}$ Manipulative Physiol Ther 37(3): 170-179.

Shumway-Cook, A. and M. H. Woollacott (2003). Controle motor: teoria e aplicações práticas, Manole.

Song, H.-S., S.-S. Woo, et al. (2014). "Effects of 16-week functional movement screen training program on strength and flexibility of elite high school baseball players." J Exerc Rehabil 10(2): 124-130.

Sorenson, E. A. (2009). Functional Movement Screen as a Predictor of Injury in High School Basketball Athletes, University of Oregon.

Sprague, P. A., G. M. Mokha, et al. (2014). "Changes in functional movement screen scores over a season in collegiate soccer and volleyball athletes." J Strength Cond Res 28(11): 3155-3163.

Steffen, K., C. A. Emery, et al. (2013). "High adherence to a neuromuscular injury prevention programme (FIFA 11+) improves functional balance and reduces injury risk in Canadian youth female football players: a cluster randomised trial." British Journal of Sports Medicine.

Teyhen, D., M. F. Bergeron, et al. (2014). "Consortium for health and military performance and American College of Sports Medicine Summit: utility of functional movement assessment in identifying musculoskeletal injury risk." Curr Sports Med Rep 13(1): 52-63.

Teyhen, D. S., S. W. Shaffer, et al. (2014). "Clinical measures associated with dynamic balance and functional movement." J Strength Cond Res 28(5): 1272-1283. 


\section{$\Psi$ Universidade de Brasília}

\section{Termo de Consentimento Livre e Esclarecido - TCLE}

O(a) senhor(a) está sendo convidado(a) a participar do projeto: "Variáveis físicas, fisiológicas e domínios de qualidade de vida: quais diferenciam os grupos classificados pelos diferentes escores do FMS?".

O objetivo desta pesquisa será verificar quais variáveis discriminam as diferentes classificações do FMS (Functional Movement Screen) em indivíduos sadios e ativos fisicamente.

O(a) senhor(a) receberá todos os esclarecimentos necessários antes e no decorrer da pesquisa e the asseguramos que seu nome não aparecerá, sendo 
mantido o mais rigoroso sigilo através da omissão total de quaisquer informações que permitam identificá-lo(a).

Você participará por meio de uma entrevista e uma avaliação inicial, na qual será verificada a presença de assimetrias posturais e condição de saúde em geral. Após essa avaliação, você será instruído verbalmente sobre todos os procedimentos do estudo e convidado a participar. O procedimento será composto por uma avaliação física e postural; avaliação do movimento por meio de análise cinemática e FMS. Você participará do projeto por aproximadamente 1 semana. A duração total de cada visita será de aproximadamente 30 minutos a 1 hora. Em cada visita, inicialmente, você deverá realizar um aquecimento leve em uma bicicleta ergométrica, de 5 a 10 minutos. Em seguida, será posicionado no equipamento correspondente à avaliação do dia. Sempre que tiver dúvidas, nós o instruiremos acerca dos requisitos (estabilização, posicionamento, cooperação, etc). Ressalta-se que todos os equipamentos de medida utilizados (dinamômetro e cinemetria) são protegidos contra descarga elétrica, não havendo riscos desta natureza. Após a calibragem dos equipamentos, você deverá realizar os testes, sendo que os resultados poderão ser visualizados em uma tela de computador à sua frente.

A possibilidade de ocorrência de problemas ou danos físicos é desprezível. No entanto, se você se sentir cansado ou desconfortável, o teste será interrompido imediatamente. Informamos também que o(a) Senhor(a) pode se recusar a responder (ou participar de qualquer procedimento) qualquer questão que lhe traga constrangimento, podendo desistir de participar da pesquisa em qualquer momento sem nenhum prejuízo para o(a) senhor(a). Sua participação é voluntária, isto é, não há pagamento por sua colaboração.

Você se beneficiará com esta pesquisa, ao ganhar avaliações posturais, de movimento e de força, além de entrar em contato co $\mathrm{m}$ equipamentos científicos modernos. Os achados da pesquisa poderão embasar cientificamente o uso do FMS em atividades desportivas e processo de reabilitação, com o intuito de melhor prever possíveis lesões musculares. Ainda, os resultados deste trabalho serão possivelmente publicados em uma revista científica. No entanto, ressaltamos que sua identidade será mantida em sigilo, e os dados serão guardados apenas pelo pesquisador responsável pelo projeto. 
Se o(a) senhor(a) tiver qualquer dúvida em relação à pesquisa, por favor telefone para: Dr. Rodrigo L. Carregaro, nos telefones: 3107-8416 ou 8119-7910, em horário comercial (das 08:00 às 12:00h e das 14:00 às 17:00h).

Este projeto foi aprovado pelo Comitê de Ética em Pesquisa da Faculdade de Ciências da Saúde da Universidade de Brasília. As dúvidas com relação à assinatura do TCLE ou os direitos do sujeito da pesquisa, podem ser obtidos através do telefone: (61) 8172-9962.

Este documento foi elaborado em duas vias, uma ficará com o pesquisador responsável e a outra com o sujeito da pesquisa. Após a leitura, o pesquisador e você deverão rubricar a primeira página e assinar a última página.

Nome / assinatura

Pesquisador Responsável

Nome e assinatura

Brasília, de de 
ANEXO II 
CRITÉRIOS DE PONTUAÇÃO DO FUNCTIONAL MOVEMENT SCREEN

\begin{tabular}{|c|c|c|c|}
\hline MOVIMENTO & III & II & I \\
\hline Deep Squat & $\begin{array}{l}\text { - Parte superior do } \\
\text { torso paralelo com a } \\
\text { tíbia ou na posição } \\
\text { vertical. } \\
\text { - Fêmur abaixo de } \\
\text { horizontal. } \\
\text { - Joelhos alinhados } \\
\text { sobre os pés. } \\
\text { - Dowel aligned over } \\
\text { feet }\end{array}$ & $\begin{array}{l}\text { * Levanta o calcanhar } \\
\text { do chão. } \\
\text { - Mesmos critérios } \\
\text { do escore III }\end{array}$ & $\begin{array}{lr}\text { - Não é } & \text { possível } \\
\text { realizar } & 0 \\
\text { movimento, mesmo } \\
\text { levantando ranoro } \\
\text { calcanhar do chão. }\end{array}$ \\
\hline Hurdle Step & \begin{tabular}{|l} 
- Quadris, joelhos e \\
tornozelos \\
alinhados no plano \\
sagital. \\
- $\begin{array}{l}\text { Postura ereta } \\
\text { sustentada. }\end{array}$ \\
\end{tabular} & $\begin{array}{l}\text { - Um ou mais } \\
\text { critérios } \\
\text { pontuação III não é } \\
\text { realizado. }\end{array}$ & $\begin{array}{l}\text { - Contato entre o pé e } \\
\text { o obstáculo. } \\
\text { - Perca de equilíbrio. }\end{array}$ \\
\hline In-line Lunge & $\begin{array}{ll}\text { - } & \text { Dowel } \\
\text { - } & \text { Dowel } \\
\text { - Joelhos tocam na } & \text { tábua. } \\
& \\
\end{array}$ & $\begin{array}{l}\text { - Um ou mais } \\
\begin{array}{l}\text { critérios } \\
\text { pontuação III não é } \\
\text { realizado. }\end{array} \\
\end{array}$ & - Perca de equilíbrio. \\
\hline Shoulder Mobility & $\begin{array}{llr}\text { - } & \text { Punhos estão } \\
\text { dentro de } & \text { um } \\
\text { comprimento } & \text { de } \\
\text { mão. } & \end{array}$ & $\begin{array}{llr}\text { - Punhos estão } & \text { um } \\
\text { dentro de } & \text { um } \\
\text { comprimento } & \text { e } \\
\text { meio de mão. } & \\
\end{array}$ & $\begin{array}{l}\text { - Punhos não } \\
\text { alcançam um } \\
\text { comprimento e meio } \\
\text { de mão. }\end{array}$ \\
\hline $\begin{array}{l}\text { Active Straight Leg } \\
\text { Raise }\end{array}$ & $\begin{array}{l}\text { - Tornozelo passa do } \\
\text { ponto médio da } \\
\text { coxa. }\end{array}$ & $\begin{array}{l}\text { - Tornozelo entre o } \\
\text { joelho e o ponto } \\
\text { médio da coxa. }\end{array}$ & $\begin{array}{l}\text { - Tornozelo não } \\
\text { passa o joelho. }\end{array}$ \\
\hline $\begin{array}{l}\text { Trunk Stability Push- } \\
\text { up }\end{array}$ & $\begin{array}{l}\text { - Homens: } 1 \text { rep; } \\
\text { polegares } \\
\text { alinhados com a } \\
\text { parte superior da } \\
\text { testa. } \\
\text { - Mulheres: } 1 \text { rep; } \\
\text { polegares } \\
\text { alinhados com o } \\
\text { queixo. }\end{array}$ & 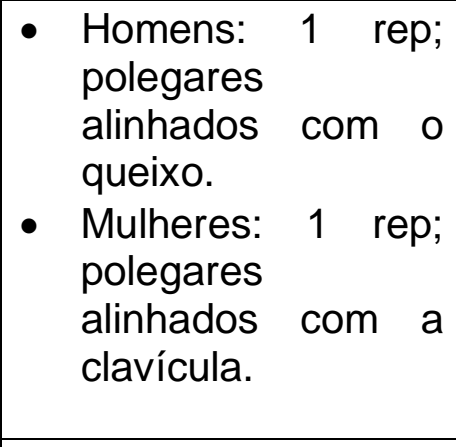 & $\begin{array}{l}\text { - Homens: incapaz } \\
\text { de atingir escore II. } \\
\text { - Mulheres: incapaz } \\
\text { de atingir escore II. }\end{array}$ \\
\hline Rotary Stability & $\begin{array}{l}\text { - Executa repetição } \\
\text { unilateral. } \\
\text { - Coluna paralela } \\
\text { com a tábua. } \\
\text { - Joelho e cotovelo } \\
\text { tocam sobre a } \\
\text { tábua. }\end{array}$ & $\begin{array}{l}\text { - Executa repetição } \\
\text { diagonal. } \\
\text { - Mesmos critérios } \\
\text { do escore III. }\end{array}$ & $\begin{array}{l}\text { - Incapaz de realizar } \\
\text { repetição diagonal. }\end{array}$ \\
\hline
\end{tabular}

${ }^{*}$ FMS criado por: Gray Cook \& Lee Burton.

** Nota: Dor $=0$ 


\section{TABELA DE PONTUAÇÃO FMS}

NOME:

IDADE:

anos PESO:

kg SEXO: ( ) M ( ) F

ALTURA: $\mathrm{m}$

ESPORTE/ATIVIDADE DE REFERÊNCIA:

\section{DOMINÂNCIA}

MÃO:（）ESQUERDA （）DIREITA PERNA:（）ESQUERDA（）DIREITA

\begin{tabular}{|c|c|c|c|}
\hline TESTE & ESCORE BRUTO & FINAL & COMENTÁRIO \\
\hline \multicolumn{4}{|l|}{ DEEP SQUAT } \\
\hline \multicolumn{4}{|l|}{ HURDLE ST. DIR. } \\
\hline \multicolumn{4}{|l|}{ HURDLE ST. ESQ. } \\
\hline \multicolumn{4}{|l|}{ IN-LINE LUN. DIR. } \\
\hline \multirow{2}{*}{\multicolumn{4}{|c|}{$\begin{array}{l}\text { IN-LINE LUN. } \\
\text { ESQ. }\end{array}$}} \\
\hline & & & \\
\hline \multicolumn{4}{|l|}{ SHO. MOB. DIR. } \\
\hline \multicolumn{4}{|l|}{ SHO. MOB. ESQ. } \\
\hline \multicolumn{4}{|l|}{ ACTIVE IMP. DIR. } \\
\hline \multicolumn{4}{|l|}{ ACTIVE IMP. ESQ. } \\
\hline \multicolumn{4}{|l|}{ ASLR DIR. } \\
\hline \multicolumn{4}{|l|}{ ASLR ESQ. } \\
\hline \multicolumn{4}{|l|}{ TSPU } \\
\hline \multicolumn{4}{|l|}{ EXT } \\
\hline \multicolumn{4}{|l|}{ ROT. STAB. DIR. } \\
\hline \multicolumn{4}{|l|}{ ROT. STAB. ESQ. } \\
\hline \multicolumn{4}{|l|}{ FLX } \\
\hline TOTAL & & & \\
\hline
\end{tabular}

